\title{
Polymorphism and phase transitions of the muscle relaxant thiocolchicoside
}

Graciela Diaz De Delgado ${ }^{1}$, Robert A. Toro H. ${ }^{1}$, José Hernando Quintana ${ }^{2}$, José Antonio Henao Martínez ${ }^{2}$, José Miguel Delgado Quiñones ${ }^{1}$

${ }^{1}$ Universidad De Los Andes, Merida, Venezuela, RB, ${ }^{2}$ Universidad Industrial de Santander, Bucaramanga, Colombia E-mail: diaz@ula.ve

The study of polymorphism, solvation, and stability, particularly in the solid state, of Active Pharmaceutical Ingredients (APIs) is of paramount importance in the pharmaceutical industry. These studies contribute to understand the behavior of the API under different synthesis, purification, storage, and handling conditions, and can ultimately determine the final commercial formulation in which the API could be marketed. Despite the vast amount of knowledge accumulated on commonly used APIs, many of them have not been reported. Over the years, we have studied a variety of APIs in order to understand their crystallization and solid state behavior. One of them is Thiocolchicoside (THC), a muscle relaxant widely used in many countries. THC is a synthetic sulfur derivative of colchicine which is isolated from Colchicum autumnale. Colchicine also has muscle relaxant activity but the presence of a glucopyranosyl group and a Sulfur atom in THC increases its therapeutic properties. It is formulated as tablets of 2 or $4 \mathrm{mg}$ or in combination with ibuprofen, acetaminophen, and other compounds with analgesic and anti-inflammatory activity. Interestingly, THC has a complex behavior in the solid state and under different crystallization conditions which has not been fully described. As shown in Figure 1, raw THC is anhydrous (Phase-1). The indexing of the powder $X$ ray diffraction pattern of raw THC resulted in an orthorhombic cell with a=28.029(7) $\AA$, $b=12.525(3) \AA, c=8.528(2) \AA$, and $V=2993.80 \AA 3$ which had not been reported before. From an aqueous solution maintained at $37{ }^{\circ} \mathrm{C}$ for 4 hours, crystals of $\mathrm{THC} .2 \mathrm{H} 2 \mathrm{O}$ were obtained. Upon grinding, this dihydrate produces an amorphous phase while dissolution in ethanol:water produces an ethanol-hydrate solvate. Additionally, heating the dihydrate at $90-120^{\circ} \mathrm{C}$ produces a second anhydrous phase (Phase-2) which, upon cooling, gives anhydrous Phase-3. A fourth phase can be obtained from Phase- 3 by evaporation of an aqueous solution $[2,3]$. The transformations that THC undergoes and the crystal structure of the dihydrate and ethanol-hydrate will be discussed in detail.

Acknowledgements. This work has been possible thanks to Grant LAB-97000821 from FONACIT-Venezuela and Grant 1518 from Vicerrectoría de Investigación y Extensión, Universidad Industrial de Santander (UIS) Bucaramanga, Colombia. R.A.Toro $\mathrm{H}$. is currently a Postdoctoral fellow at Universidad Industrial de Santander, Bucaramanga, Colombia.

[1] Cui, Y. (2007). Inter. J. Phar. 339, 3-18.

[2] Toro H., R. A. (2015) Ph.D. Thesis. Universidad de Los Andes, Mérida, Venezuela.

[3] Toro, R. et al. (2014) Powder Diffr. 29, 62-68.

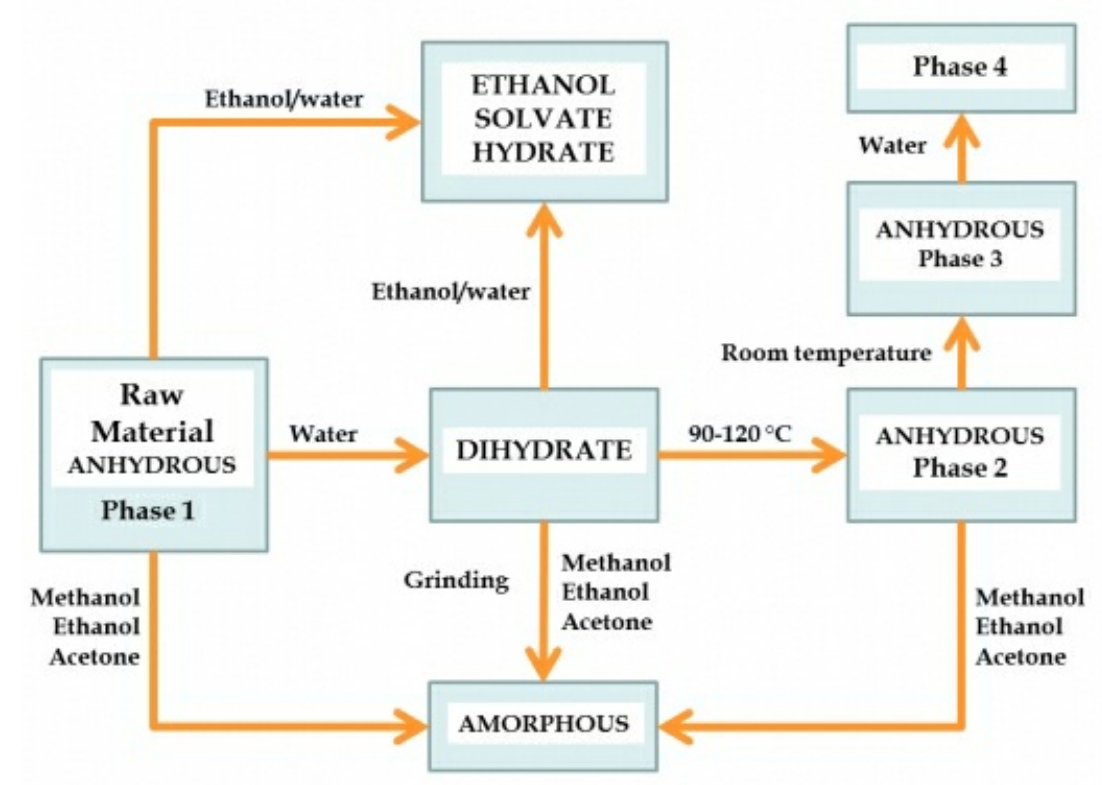

Keywords: Thiocolchicoside, muscle relaxant, API 